\title{
Ring testing of the AnBUSDiC test; a new screening method for the anaerobic degradability of surfactants
}

\author{
Charles Eadsforth ${ }^{1}$, Tom Austin ${ }^{1}$, Alain Bouvy², Giorgio Cassani ${ }^{3}$, Juanantonio DeFerrer ${ }^{4}$, Claus-Dierk Hager ${ }^{5}$, \\ Diederik Schowanek ${ }^{6}$, Klaus Taeger ${ }^{7}$ and Andreas Willing ${ }^{8^{*}}$
}

\begin{abstract}
Background: Surfactants are widely used across the globe both in industrial and consumer products; their biodegradation characteristics are therefore of high importance. Upon entering a wastewater treatment plant (WWTP), the majority of surfactants are aerobically mineralized to $\mathrm{CO}_{2}$ and $\mathrm{H}_{2} \mathrm{O}$. However, a small fraction is inevitably left non-degraded and adheres to the remaining sludge. This sludge is usually further treated in anaerobic digester tanks. Assessment of existing methods for determining anaerobic biodegradability has led to the development of a new test method, which is in principle based on the method DIN 38414 part 8 . This new test, named the anaerobic biodegradation under sludge digester conditions test (abbreviated to AnBUSDiC test) allows for a quantification of the degradation of surfactants under conditions encountered in the anaerobic digester tank of municipal WWTPs. The AnBUSDiC test has several advantages over existing methods. The main advantage is that it is particularly suitable for surfactants, because the two-step design minimizes possible unspecific digester gas formation caused by the surface activity of the test substances, therefore avoiding false positive results.

Results: In order to further standardize the AnBUSDiC test and gain regulatory acceptance, a ring test was organized involving seven laboratories, and five model surfactants from different surfactant classes (anionic, nonionic (branched and linear) and amphoteric) plus a positive control, glucose. The AnBUSDiC test produced reliable repeatable results between laboratories; however, some additional modifications were suggested. It was identified that the original test method did not identify a clear endpoint from which a biodegradation value should be taken. It was proposed that a new more concise endpoint be defined in combination with the AnBUSDiC test to allow better comparability between test results.
\end{abstract}

Conclusions: The inclusion of a second addition of test substance is a major step forward in the elimination of the variability produced by non-specific gas production. With the exception of one anomalous result for linear alkylbenzene sulfonates, for which an explanation can be provided, the AnBUSDiC method appears to provide overall robust and interpretable results.

Keywords: Surfactants; Anaerobic biodegradation; Environmental fate; Digester tank simulation test; Ring test

\footnotetext{
* Correspondence: andreas.willing@basf.com

${ }^{8}$ BASF Personal Care and Nutrition GmbH, Henkelstr 67, Dusseldorf 40589,

Germany

Full list of author information is available at the end of the article
} 


\section{Background}

Surfactants are widely used across the globe in both industrial and consumer products, so their biodegradation characteristics are therefore of high importance. The biodegradability of a substance is a key parameter when considering the environmental implications of its use [1]. The fate of most surfactants is to be discharged in wastewater, usually entering a wastewater treatment plant (WWTP). Upon entering a WWTP a fraction of the surfactants, due to their surface-active nature, is adsorbed to the primary sludge. The remainder is almost completely mineralized to $\mathrm{CO}_{2}$ and $\mathrm{H}_{2} \mathrm{O}$ in the aerator. The sludge containing the non-degraded surfactants is then further treated in anaerobic digester tanks. Currently, scientifically appropriate simulation methods for the analysis of surfactants in anaerobic conditions within a WWTP (OECD 314 [2]) require radiolabeled test substances, which limit the scope of such a method.

The European Commission, in a communication to the European Parliament and the Council (http://eur-lex.europa.eu/LexUriServ/LexUriServ.do?uri=COM:2009:0230: FIN:en:PDF) indicated their intention to undertake further research on the anaerobic biodegradability of surfactants as required by Article 16(2) of Regulation (EC) No. 648/2004. In response to this ERASM informed the Commission of their initiative to explore an improved method for measuring anaerobic degradability under sludge digester conditions. Assessment of existing methods for anaerobic biodegradability [3] led to the development of a new, improved test method, which is in principle based on the method DIN 38414 part 8 [4]. The newly developed test method, named the Anaerobic Biodegradation Under Sludge Digester Conditions test (or abbreviated the AnBUSDiC test [5]) overcomes the limitation of DIN 38414 part 8 in two ways:

(1) A well characterized amount of surfactant is added to the test system instead of the unknown test effluent to allow determination of an exact degradation rate

(2) A two-step addition of the test substance is introduced to avoid false positive results due to unspecific (excess) gas formation caused by the surface activity of the test substance.

The new AnBUSDiC test allows for a quantification of the degradation of surfactants under conditions encountered in the anaerobic digester tank of municipal WWTPs. The advantages of the AnBUSDiC test are that it is particularly relevant for assessment of the environmental fate of surfactants, as surfactants usually end up in the digester tank of WWTPs and the two-step design minimizes possible unspecific digester gas formation caused by the surface-activity of the surface-active test substances, thereby avoiding false positive results (i.e. the formation of biogas from unspecified substances released from the sludge rather than from the added test compound). The AnBUSDiC test conditions are closer to a simulation test rather than a screening test and it is also capable of identifying whether a surfactant is degradable, potentially non-degradable or inhibitory to the anaerobic microorganisms (i.e. preventing or reducing biodegradation in WWTP conditions).

In order to further standardize the AnBUSDiC test and gain regulatory acceptance, a ring test was organized involving seven laboratories, the results of which are presented and discussed here.

\section{Results and discussion \\ Results}

Typical gas production curves for the positive control glucose are shown in Figure 1. As detailed in the original test method [5], the majority of the unspecific sludge gas production, as demonstrated by an initial gas formation, occurs during the first 21 days (phase 1). Phase 2 is therefore more representative of the true test substance biodegradation, and the initial gas production is therefore slightly lower (Figure 1).

The associated total biodegradation curves for the positive control glucose are shown in Figure 2. The total biodegradation curve is produced (using Equation six, see [5]) based on gas production data as well as the theoretical gas production of the test substance. In some cases, negative biodegradation values can be achieved because of the comparison between test substance biodegradation and blank digester biodegradation (as shown in Figure 3). This is discussed later.

An example of negative biodegradation values for the anionic surfactant LAS are shown in Figure 3. This occurs because of the comparison between test substance biodegradation and blank digester biodegradation. Biodegradation results (as \%) for individual test substances are tabulated in Table 1 and displayed with mean \pm standard deviation in separate bar charts in Figure 4. Where possible, the standard deviation of the replicates within each lab were included in Table 1 . In general, the reproducibility within each lab was good, particularly when testing glucose or betaine. The highest standard deviation across labs was 4.7 for glucose and 8.7 for betaine. Labs 1, 3, 4 and 5 had fairly low standard deviations across the majority of test substances demonstrating good consistency between replicates. However, each lab bar, lab 4 had one test substance with a higher standard deviation value, indicating greater variability. These were results for the isoFA + 7EO, FAS and LAS test substances from labs 1,3 and 5 , respectively. When assessing all the results together, two out of the six test substances had a standard deviation of less than 10\%, 


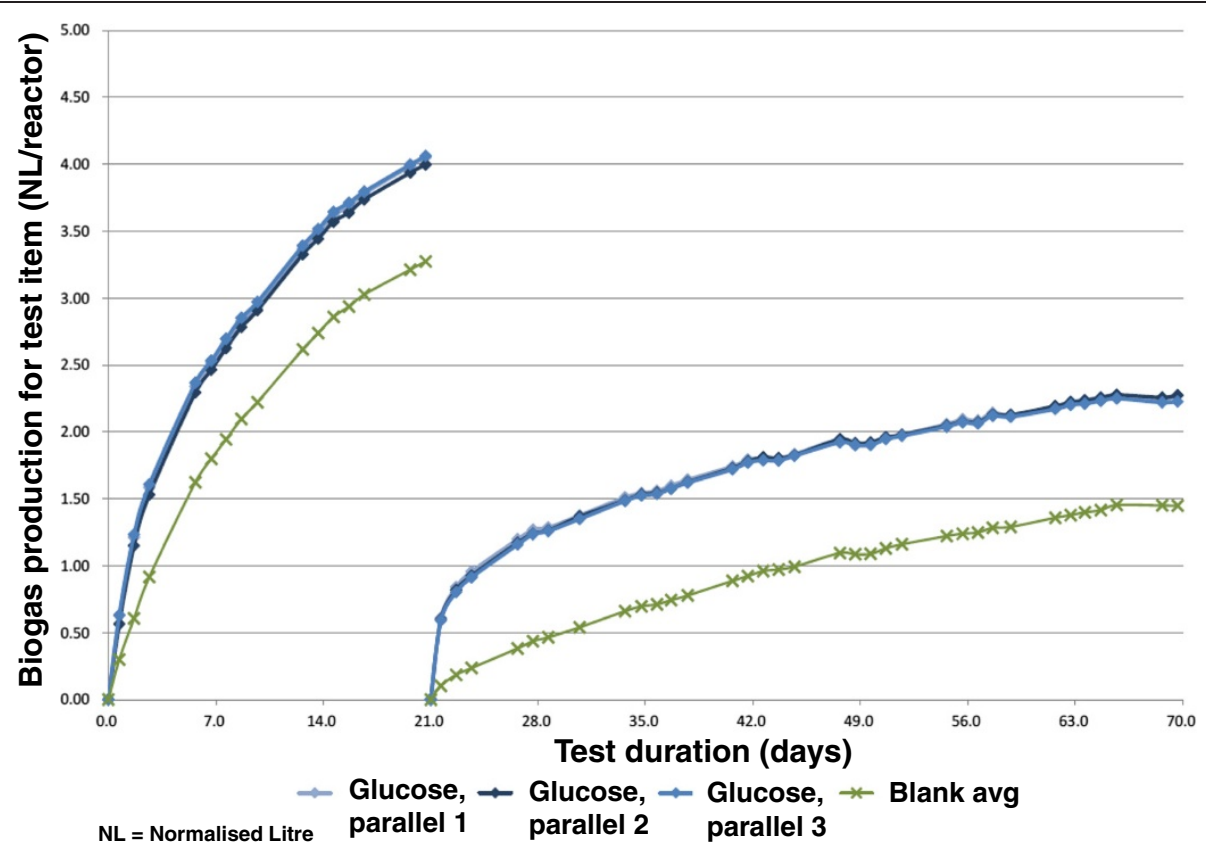

Figure 1 Representative example of total biogas production curve of glucose (positive control) compared with blank sewage sludge. Taken from a participating laboratory report.

three test substances had standard deviations of around $20 \%$ to $30 \%$ and one substance (LAS) showed highly variable results with a standard deviation of around $40 \%$. Some individual values appeared particularly erroneous; the significance of these is discussed later.
Based on the full sets of results from the participating laboratories, the two substances with the best reproducibility between labs were glucose and betaine. The positive control, glucose, achieved a mean biodegradation of $81.2 \%$ with a standard deviation of $8.8 \%$, whereas betaine

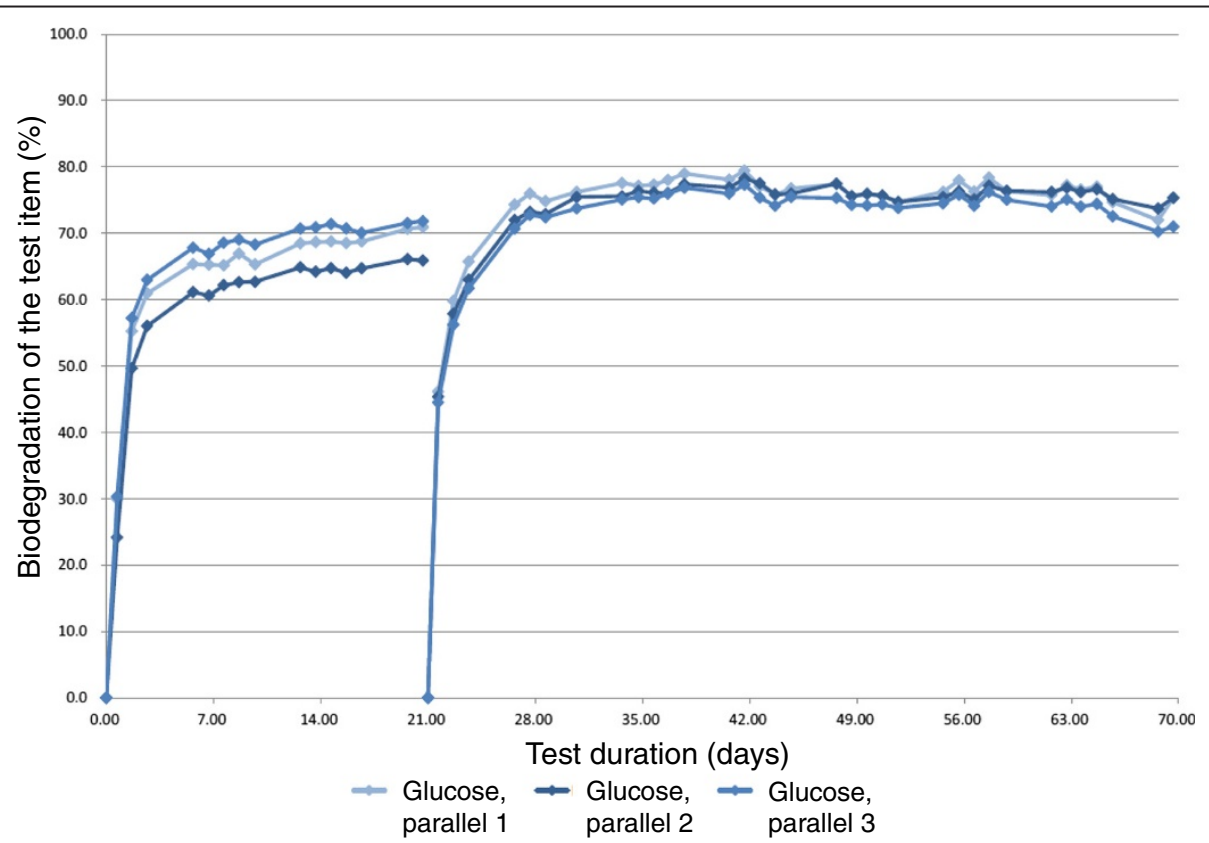

Figure 2 Representative example of a biodegradation evolution curve for glucose (positive control). Taken from a participating laboratory report. 


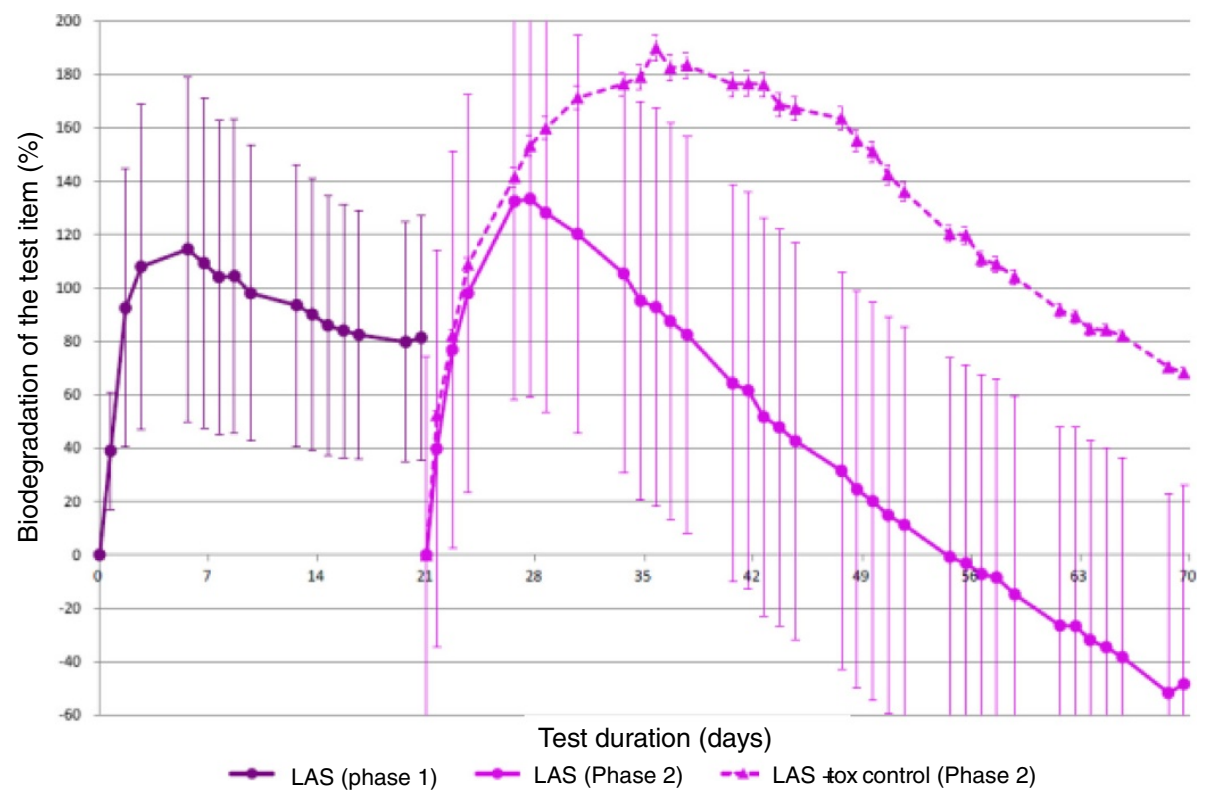

Figure 3 LAS biodegradation curve from a participating laboratory report.

achieved a mean biodegradation of $73.3 \%$ with a standard deviation of $9.8 \%$. After reaching a maximum degradation, the degradation of glucose and betaine both proceed to 'plateau' as expected.

There were also two substances with higher degradability results than the positive control, glucose. FA + 7EO achieved a mean biodegradation of $97.1 \%$ with a standard deviation of $26.0 \%$. This test substance exhibited a rapid initial degradation reaching its maximum (of 97.1\%), at which point the degradation curve plateaued briefly before beginning to slowly decrease. FAS achieved a mean biodegradation of $93.3 \%$ with a standard deviation of $31.2 \%$. After reaching its maximum degradation (of 93.3\%) the degradation curve plateaued. One of the laboratories suggested that FAS may have shown some initial toxic effects at the tested concentration as the degradation of the toxicity controls were significantly lower than the test substance alone. However, the FAS degradation levels were sufficiently high to suggest that the anaerobic degradation of the test material had not been significantly affected after 60 days.

Both these high anaerobic degradation results were achieved despite the inclusion of potentially anomalous results of $38 \%$ and $37 \%$, respectively, for these substances which were both generated by laboratory 3 . Laboratory 3 reported a toxic influence of test substance FAS on the inoculum which explains the lower biodegradation value compared with other labs; however, this is not the case for FA + 7EO. It is noted that this laboratory also generated significantly lower biodegradation levels for a number of test substances when compared with other labs. Conversely, the degradation of the

Table 1 Percentage biodegradation results, with mean, standard deviation and $95 \%$ confidence interval

\begin{tabular}{|c|c|c|c|c|c|c|}
\hline \multirow[t]{2}{*}{ Laboratory } & \multicolumn{6}{|c|}{ Biodegradation (\%) (+/- SD, where available) <lag period in days $>$} \\
\hline & Glucose & Betaine & $\mathrm{FA}+7 \mathrm{EO}$ & FAS & isoFA + 7EO & LAS \\
\hline 1 & $81.9(4.7)<0>$ & $76.8(4.75)<0>$ & $95.3(2.88)<0>$ & $97.4(7.29)<0>$ & $66.3(30.7)<39>$ & $22.7(1.66)<0>$ \\
\hline 2 & $69.0<0>$ & $72.0<0>$ & $103.0<0>$ & $65.0<35>$ & $98.0<0>$ & $0.0<105>$ \\
\hline 3 & $76.0(4)<0>$ & $53.0(5.51)<0>$ & $38.0^{\mathrm{a}}(11.02)<0>$ & $37.0^{\mathrm{a}}(48.64)<39>$ & $1.3^{\mathrm{a}}(1.53)<40>$ & $0.0(1.15)<40>$ \\
\hline 4 & $92.7(3.51)<0>$ & $77.0(2.83)<0>$ & $106.0(15.56)<0>$ & $123.0(8.49)<0>$ & $51.5(0.71)<0>$ & $31.0(2.83)<0>$ \\
\hline 5 & $79.0(\mathrm{NA})<0>$ & $83.7(8.7)<0>$ & $95.9(1.3)<0>$ & $117.6(3.4)<0>$ & $51.2(1.4)<0>$ & $133.4^{a}(62)<0>$ \\
\hline 6 & $74.9<0>$ & $67.3<0>$ & $123.4<0>$ & $84.0<4>$ & $23.9<33>$ & $6.2<0>$ \\
\hline 7 & $95.0<0>$ & $83.0<0>$ & $118.0<0>$ & $129.0<0>$ & $55.0<0>$ & $26.0<0>$ \\
\hline Mean (+/- SD) & $81.2(8.8)$ & $73.3(9.8)$ & $97.1(26.0)$ & $93.3(31.2)$ & $49.6(28.5)$ & $31.3(43.3)$ \\
\hline${ }^{\mathrm{b}}$ Mean (+/- SD) & & & $106.9(10.5)$ & $102.7(22.8)$ & $57.7(22.1)$ & $14.3(12.7)$ \\
\hline
\end{tabular}

${ }^{\mathrm{a}}$ Questionable result based on the reported anaerobic biodegradability of this type of surfactant, e.g. according to the DID list; ${ }^{\mathrm{b}}$ Mean and standard deviation calculated excluding anomalous result $\left({ }^{(}\right)$. 


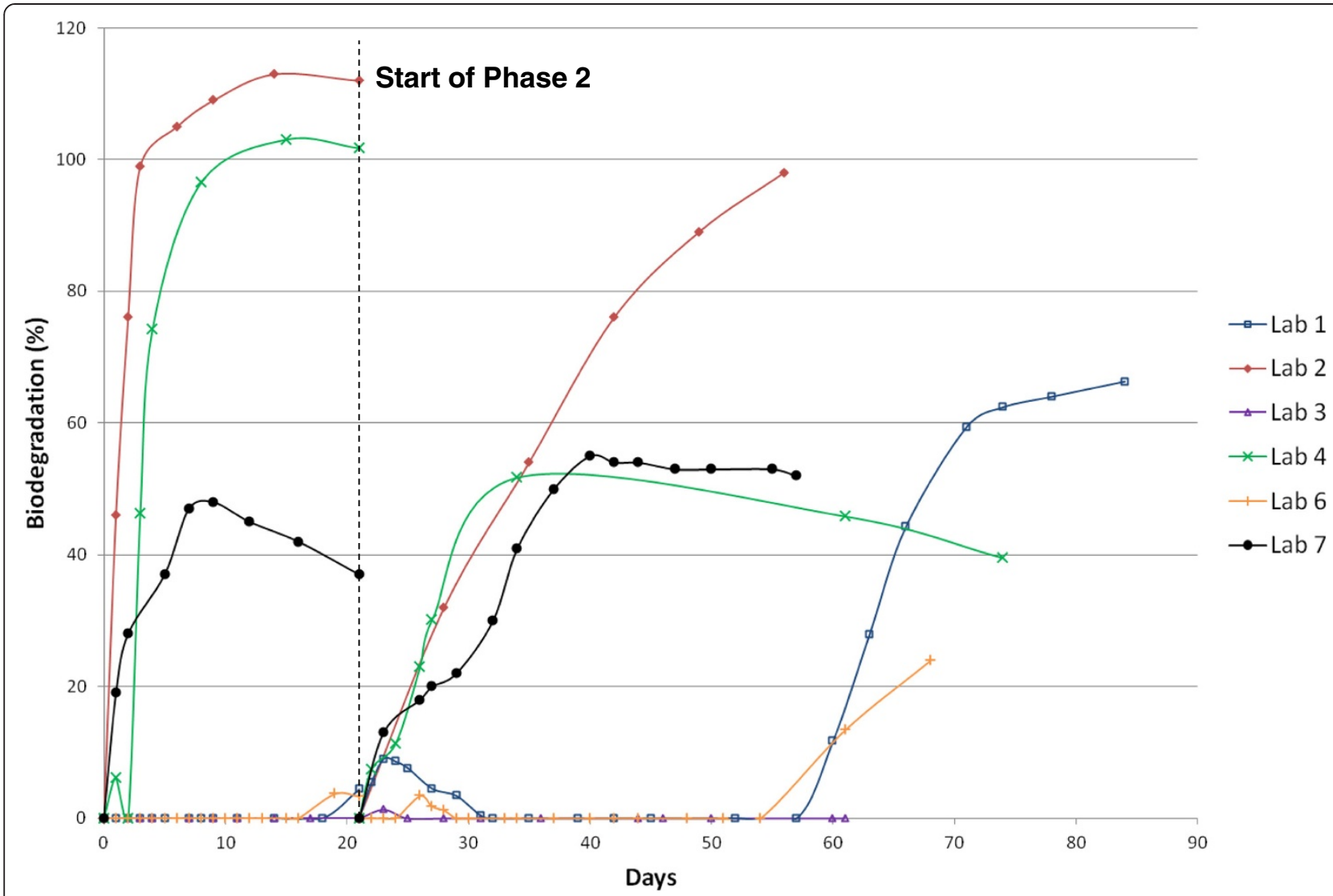

Figure 4 Test substance degradation values obtained by participating laboratories. Mean and standard deviation shown. All data included.

positive control substance glucose is within acceptable limits, and therefore, it must be summised that the low value for FA + 7EO and the toxicity demonstrated by FAS was simply a result of the inherent variability between inoculum sources. If these results are discounted, the results show much less variation with lower standard deviations. FA + 7EO achieves a mean degradation of $106.9 \%$ and standard deviation of $10.5 \%$, and FAS achieves a mean degradation of $102.7 \%$ and standard deviation of $22.8 \%$.

The results of the remaining two substances were considerably influenced by two potentially anomalous results. The test substance isoFA + 7EO achieved a mean degradation of $49.6 \%$ with a standard deviation of $28.5 \%$ and, in particular, showed inconsistent degradation patterns amongst some of the participating laboratories. During phase 2, testing isoFA + 7EO exhibited a lag phase of between 20 and 32 days in three different laboratories. The large lag phase meant that laboratory 6 potentially did not continue the study to the maximum achievable level of degradation and so would have returned a lower result. On termination of phase 2 , the percentage degradation of isoFA + 7EO was still increasing, indicating that a plateau would have been reached given enough time. Additionally, laboratory 3 only experienced minimal (i.e. 1\%) anaerobic degradation of isoFA + 7EO and reported that this test substance demonstrated toxicity towards the inoculum. In two of the remaining laboratories, isoFA + 7EO did not exhibit a lag phase; however, the toxicity control degradation levels at the end of phase 2 were significantly lower, indicating either a toxic or inhibitory effect. If the result from laboratory 3 is discounted, then the mean degradation value for isoFA + 7EO was $57.7 \%$ with a standard deviation of $22.1 \%$. The biodegradation curves of isoFA + 7EO are displayed in Figure 5.

LAS achieved a mean degradation of $31.3 \%$ with a standard deviation of $43.3 \%$. All but one laboratory achieved degradation in the range $0 \%$ to $31 \%$, with laboratory 5 achieving a much higher anomalous value of $133.4 \%$. If the anomalous result is discounted, then the mean degradation value for LAS was $14.3 \%$ with a standard deviation of $12.7 \%$. In the LAS studies, the initial gas formation in phase 1 proceeded normally. However, after a few days of normal degradation in phase 2 , there was a severe decline in the degradation rate of LAS resulting in a downward sloping degradation curve producing negative values (as shown in Figure 3). In addition, the toxicity control also follows a similar pattern with a 

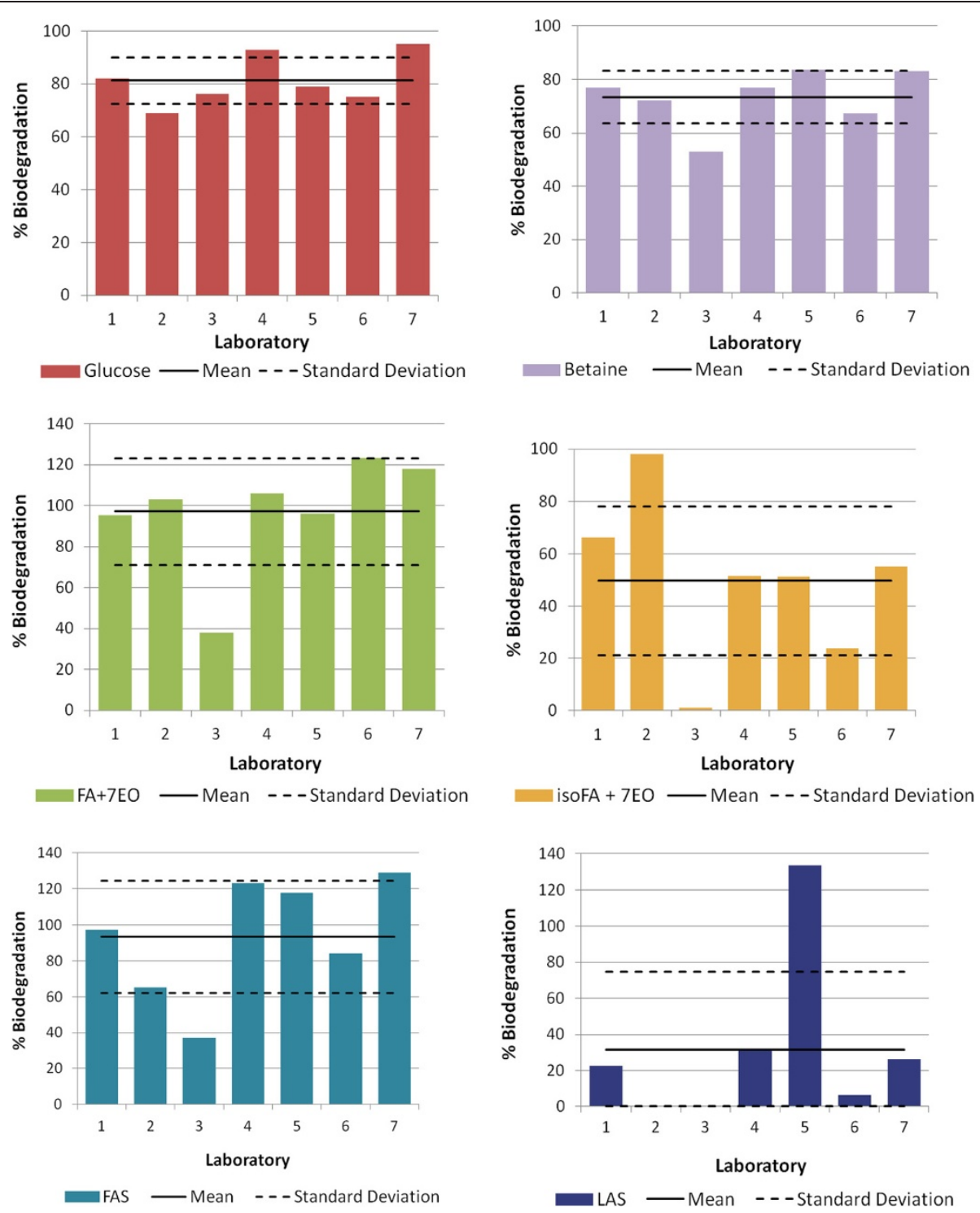

Figure 5 Mean \% biodegradation of isoFA + 7EO comparing the variation in lag phases.

small lag. These results indicate some form of inhibitory action by LAS at the tested concentrations. Total biodegradation results (as \%) for the isoFA + 7EO test substance are tabulated in Table 1. Individual test substance degradation values (mean and standard deviation) are shown in Figure 4.

\section{Discussion}

Using the revised AnBUSDiC test procedure [5], there were noticeable differences in the levels of anaerobic biodegradation observed for the six different test substances. When all data submitted by the participating laboratories were included, four of the test substances, FA + 7EO (a non-ionic linear alcohol ethoxylate), FAS (the anionic sodium dodecyl sulphates), betaine (an amphoteric surfactant) together with the positive control (glucose) were all efficiently anaerobically degraded with mean degradation levels of more than $70 \%$. Possible anomalous (lower biodegradation percentage) results were observed from participating laboratory 3 for FA $+7 E O$ and FAS. Exclusion of these two values resulted in higher degradation and improved standard deviation values for these two test products but does not change the overall conclusion that both these products are efficiently anaerobically degraded. Whilst the degradation of these two substances in laboratory 3 was lower compared with the other labs, degradation of the positive control substance glucose was within the normal range, and therefore, it must be summised that the low values are simply a result of the inherent variability 
between inoculum sources ability to degrade the test substances. When previously tested under OECD 311 test guidelines, FA + 7EO was found to be $70 \%$ to $80 \%$ biodegradable in 60 days which supports the ring test data found in this study [1].

The test substance isoFA + 7EO (a nonionic branched alcohol ethoxylate) was anaerobically degraded slightly less (i.e. 50\%) than its linear equivalent (FA + 7EO) when all the data were included. Exclusion of the lower biodegradation value (1\%) submitted by laboratory 3 , resulted in a higher degradation (58\%) and improved standard deviation value for isoFA + 7EO. IsoFA + 7EO appeared to show a certain amount of persistence; most laboratories experienced a lag phase of some length during which the inoculums adapted to the substrate. Toxic controls were not affected, indicating the test item was not poorly degraded because of toxicity. The lag phase indicates an initial period whereby microorganisms needed to adapt to the presence of isoFA + 7EO. This initial lag phase and lower overall degradation (compared with the linear FA + 7EO) was likely a result of the increased resistance to biodegradation under aerobic and anaerobic conditions of hydrocarbons with iso branching [6,7]. Each test assay was subject to inherent variability brought about by the various sludge inoculum sources. Each sludge may have been exposed to differing wastewater conditions resulting in a variety of microbial communities with differing abilities to adapt to each test substance. All tests were considered valid, with the sludge blanks producing gas within the required range of 1.2 to $3 \mathrm{NL} / \mathrm{Kg}$. The lower degradation value for the branched isoFA + 7EO in comparison to that for the linear FA + 7EO can be attributed to the effect of quaternary carbon atoms in the alkyl chain, an effect which is also seen in aerobic degradation [6] and anaerobic degradation studies [6]. The isoFA + 7EO data are consistent with those reported by Berna et al. [1].

The anionic surfactant LAS was degraded much less than the other surfactants. It achieved a mean degradation of $31.3 \%$ with a standard deviation of $43.3 \%$ when all the test data were included. The high mean degradation and standard deviation values are due to a single anomalous degradation value of $133.4 \%$ submitted by laboratory 5. If the anomalous result is discounted, then LAS achieves a mean degradation of $14.3 \%$ with a standard deviation of $12.7 \%$. Laboratory 5 found no toxic effects associated with LAS as demonstrated by the toxicity control. However, it was noted that the results appeared 'strange', although no problems were found. Possible explanations might be (a) the presence of oxygen in the system (see below) or (b) that the observed 'degradation' was an artifact due to a not properly sealed apparatus and/or that the bottles containing the excess eudiometer solution were not placed at about the same height as the meniscus of the liquid inside the eudiometer (see [5] for a more detailed explanation).

The graph produced (Figure 3 ) shows that after an initially high biodegradation rate, biodegradation of the test substance decreases dramatically resulting in a downward sloping curve (most likely because most of the test substance has been degraded at this stage). The other six laboratories found that LAS did not degrade at all or that only a very low percentage was degraded and that the biological activity of the sludge had been inhibited. Most laboratories found that the phase 1 LAS degradation proceeded normally, however, after around ten days into the second phase an early plateau was reached, leading into a decreasing degradation curve, the same also happened with the toxicity/inhibition control. A possible explanation is that after only being degraded by around 50\% during the first phase, the second addition of test substance exceeded a concentration threshold, over which LAS is inhibitory or toxic to the inoculum. These results agree with the majority of the scientific literature on the subject of anaerobic LAS biodegradation, it has been found that over a certain concentration LAS can become inhibitory to biogas production; this threshold has been shown to be as low as just 20 ppm [1]. It has only recently been shown that the full anaerobic biodegradation of LAS is possible. However, it appears very specific to certain microorganisms and has only been demonstrated in marine sediment studies [8]. In addition, the average half-life of LAS in this study was 90 days, making this an unlikely explanation for this result. It is more likely that a small amount of oxygen was present in the experimental system; work with sulfonate such as LAS has shown that once sulfonate biodegradation has been initiated in oxygen-limited systems, the intermediates can continue to degrade anaerobically [1]. In addition, the results of laboratory 5 did not show LAS had any toxic/inhibitory effects, which would be the case if it was aerobically degraded quickly. The AnBUSDiC test appears capable of demonstrating degradability of test items with inhibitory and toxic effects provided a suitable concentration is used; LAS has been previously shown not to degrade anaerobically to biogas in other tests [1]. It can therefore be concluded that LAS is unlikely to undergo high levels of biodegradation within the anaerobic compartments of a WWTP.

The amount of variation between laboratories in this ring test is considered normal when compared with previous test results from both aerobic and anaerobic ring tests [9]. It is apparent that laboratory 3 appeared to suffer consistently lower results for the test substances compared to the other laboratories, which suggests that the sludge inoculum may have been less active. The AnBUSDiC test shows a greater degree of reproducibility than the DIN 38414 part 8 and ISO 11734 anaerobic 
methods $[4,5,9]$. Issues during the DIN 38414 part 8 test with unspecific sludge degradation leading to degradation values over $100 \%$ were to a large extent solved with the two phase test design of the AnBUSDiC test. However, mean degradation values of over $100 \%$ were still encountered in the second test phase with FA+7EO and FAS, albeit to a lesser extent. The 1st phase addition of test substance appears to have released most of the organic carbon 'trapped' in the sludge (via the solubilising action of the surfactant test item), the two phase design was implemented to reduce this unspecific degradation during the second phase addition (however, some still occurs) [5]. Using a two-phase addition of test substance (rather than three dosings) was deemed a compromise between the test length and the potential to overestimate test substance degradation. It was therefore expected that highly degradable test substances would display values close to or over 100\%; however, values in some tests did exceed $120 \%$. When developing the AnBUSDiC test it was clear that the two phase test did not totally eliminate the release of organic carbon from the sludge during the second addition; however, values over $120 \%$ were not expected anymore.

An explanation for the overestimation of the degradation value is that the total organic carbon (TOC) value used to calculate the theoretical gas production (using Equation one from Bendt and Willing [5]) is too low and does not represent the TOC left in the system in phase 2. There was a discussion within Bendt and Willing [5] with regards to the calculation of the maximum theoretical gas production value if $100 \%$ of the test substance TOC was to be degraded. It was argued that the TOC used in the calculation of the theoretical gas production should be taken as the TOC from the second addition of test substance only. The justification was that when phase 1 reaches the plateau phase the remaining test substance is no longer degradable and that the addition of the remaining phase 1 TOC to the second phase TOC (for use in calculating theoretical gas production) would lead to underestimation of the test substance degradability (i.e. a theoretical gas production value which was too large). It is suggested that in the degradation rate graph, the plateau phase does not equate to the end of test substance degradation, but rather that the degradation of sludge with and without test substance is the same (as defined by Equation four). Achieving results of up to $130 \%$ degradation during the ring tests suggests that the degradability of test substances is being to a certain extent overestimated.

In an attempt to resolve this issue, some of the percentage biodegradation results were recalculated. A number of phase 2 biodegradation results reached over $100 \%$ biodegradation, as discussed this was partly due to an underestimation of the TOC left in the system when calculating the theoretical gas production (using Equation one from Bendt and Willing [5]). The solution suggested involved calculating the remaining TOC after phase 1 and adding it to the TOC introduced to the system in phase 2 . A brief exercise was carried out where possible to test whether using an altered TOC value in the theoretical gas production calculation would offer more realistic biodegradation values. The percentage biodegradation at the end of phase 1 was read from the original results and used to calculate the TOC non-degraded in phase 1. The remaining TOC from phase 1 was then added to the TOC added to phase 2. This value was then used in Equation one to calculate the theoretical gas production. As informed by Equation two, a higher theoretical gas production results in a lower phase 2 biodegradation percentage for the same net gas production.

As can be seen in Table 2, the altered calculation did reduce the percentage biodegradation for a selection of results. However, the results that this alteration was intended to effect could not be recalculated. In many cases where the phase 2 biodegradation was significantly over $100 \%$, it was found that the phase 1 biodegradation also exceeded $100 \%$. This meant that in theory, no leftover TOC could be added to the phase 2 total, and therefore, the results could not be recalculated. It would

Table 2 Phase $2 \%$ biodegradation results with recalculated values (where this was possible)

\begin{tabular}{|c|c|c|c|c|}
\hline \multirow[t]{3}{*}{ Test substance } & \multicolumn{4}{|c|}{ Result } \\
\hline & \multicolumn{4}{|c|}{ Lab } \\
\hline & 1 & 4 & 6 & 7 \\
\hline Glucose & 81.9 & 92.7 & 74.9 & 82.1 \\
\hline \multicolumn{5}{|l|}{ Phase 2} \\
\hline Phase 2 re-calculated & 63.2 & NA & 37.4 & 82.1 \\
\hline FAS & 97.4 & 123.0 & 84.0 & 129.0 \\
\hline \multicolumn{5}{|l|}{ Phase 2} \\
\hline Phase 2 re-calculated & NA & NA & NA & NA \\
\hline \multicolumn{5}{|l|}{ Betaine } \\
\hline Phase 2 & 76.8 & 77.0 & 67.3 & 83.0 \\
\hline Phase 2 re-calculated & 56.6 & NA & 54.8 & 71.2 \\
\hline \multicolumn{5}{|l|}{ isoFA + 7EO } \\
\hline Phase 2 & 66.3 & 51.5 & 23.9 & 55.0 \\
\hline Phase 2 re-calculated & 36.5 & NA & 12.2 & 35.5 \\
\hline \multicolumn{5}{|l|}{$\mathrm{FA}+7 \mathrm{EO}$} \\
\hline Phase 2 & 95.3 & 106.0 & 123.4 & 118.0 \\
\hline Phase 2 re-calculated & NA & NA & NA & 98.3 \\
\hline \multicolumn{5}{|l|}{ LAS } \\
\hline Phase 2 & 22.7 & 31.0 & 6.2 & 26.0 \\
\hline Phase 2 re-calculated & 16.2 & 26.7 & 3.1 & 16.0 \\
\hline
\end{tabular}

$\mathrm{NA}=$ Result could not be re-calculated as the phase 1 degradation was more than $100 \%$. 
appear that the only way to reduce the results to below $100 \%$ would be to include a third phase; however, this is impractical [5]. Whilst the alternative calculation worked in some cases, it could not be applied every time therefore in the interests of consistency should not be used. Other options may include extending phase 1 to allow further degradation of the released sludge material to prevent overestimation of the degradation in phase 2 .

Degradation percentage curves for some test substances show that after an initial peak of intense biodegradation the biodegradation curve (e.g. for FA + 7EO) then begins to decrease after a short plateau. The initial peak is likely just a large increase in degradation because of a large amount of new substrate. This initial burst of degradation caused by test item addition will leave a lower amount of degradable material within the sludge. The lower degradation rate enforced would ordinarily lead to a leveling off as seen in the gas production curves; however, when put into Equation four with the now relatively higher degradation of the blank (which did not experience the initial degradation spike and continues to increase), a negative value is obtained leading to a downward sloping curve (as shown in Figure 3). The results taken to calculate the mean were therefore taken from the maximum degradation at the beginning of the plateau or downwards curve.

During analysis of the results from the participating laboratories, it was clear that the laboratories interpreted the end point of the test in different ways. Currently, the method states that the tests are continued until the net gas production has reached the plateau phase or the degradation has reached more than $80 \%$ of the theoretical amount. However, some laboratories continued each test only to a set time limit, whereas some continued way past a plateau had been reached resulting in a downward-sloping curve. When analyzing the results, it therefore had to be decided which biodegradation value to take to compare between labs. It was decided that the value used in comparison would be the maximum value at the initial stages of the plateau. To prevent this dilemma for potential future users of the method, it should be clearly stated that the final biodegradation value should be taken as the maximum value of the first three results within $5 \%$ of each other at plateau phase (as opposed to any initial lag phase).

\section{Conclusions}

The AnBUSDiC test has been shown to produce reliable and repeatable results between laboratories. The inclusion of a second addition of test substance is a major step forward in the elimination of the variability produced by non-specific gas production. With the exception of one anomalous test result for LAS, for which a possible explanation has been provided, the method appears to provide robust and interpretable results.

Ring testing of other anaerobic biodegradation methods such as the European Centre for Ecotoxicology and Toxicology of Chemicals (ECETOC) test [9] used a validity criterion of more than $40 \%$ biodegradation to exclude anomalous results produced by poor test conditions (except where toxic/inhibitory effects were identified). Application of this criterion to this set of results identifies a number of anomalous results that might be eliminated as a result of substandard test conditions. In addition to the results discussed, applying this criterion would also remove the result for isoFA + 7EO (of 23.9\%) from laboratory 6 . Whilst this result was not identified as anomalous by the laboratory, it is over a factor of 2 lower than the mean result from the other laboratories. In the absence of any other scientific observations, this result could indicate poor test conditions, e.g. a sludge inoculum with a microbial community poorly suited to the degradation of this substance; however, this is an uncontrollable variable which contributes to the overall variation associated with this test method. It is also of note that the ECETOC validity criterion was used to exclude anomalous values for readily degradable substances only and therefore cannot be applied here regardless.

A biodegradable criterion of more than $60 \%$ degradation is recommended based on the results of substances known to be anaerobically biodegradable to biogas under sludge conditions, e.g. glucose and the alcohol ethoxylates. Additionally, a partially degradable criteria is recommended for substances which degrade in the range more than $20 \%$ to less than $60 \%$; this is based on the results of substances known to be partially degradable, e.g. isoFA + 7EO. Substances that achieve less than $20 \%$ degradation shall be regarded as non- or poorly degradable under these particular anaerobic conditions. This criterion is set based on the results of substances known to be poorly degradable in anaerobic conditions, e.g. LAS.

The anaerobic results for LAS suggest that false positives can still occur when carrying out a single test (as opposed to a ring test); however, no criteria can eliminate experimental error completely. LAS has been previously shown not to degrade anaerobically in other tests [1] and LAS has subsequently been used as a negative control substance in the AnBUSDiC test.

It was identified that the original test method did not identify a clear endpoint from which a biodegradation value should be taken. It was proposed that a new more concise endpoint be defined to allow better comparability between test results: the final biodegradation value should be taken as the maximum value of the first three results within $5 \%$ of each other at plateau phase (as opposed to any initial lag phase). 
The inclusion of non-degraded TOC from phase 1 has been discussed. Despite the addition of a second phase to account for unspecific gas production, the degradation test items still appears to be overestimated in some cases. May it be that the second addition of test substance is not sufficient to eliminate unspecific digester gas formation completely (can be checked by a third addition) or that initial inhibition (i.e. test substance gas evolution curve is temporarily below the control gas curve) leads to a carry-over of easily degradable organic matter, resulting in an overestimation of the degradation rate in the second phase, due to an underestimation of the theoretical gas production reference value. To rectify this issue, the results from some studies were recalculated with the addition of the remaining TOC from phase 1. However, it was found that this addition could not be carried out consistently and therefore did not provide a suitable solution. Whilst a small number of results for FAS and FA + 7EO were greater than $100 \%$ in phase 2 , this did not happen in every lab. One possible explanation is that the degradable material contained within the raw sludge used to create the inoculum was greater in these cases. A possible solution to this problem would be to extend the duration of phase 1 where degradation levels close to or above $100 \%$ are initially reached in phase 1 . This would allow the removal of further degradable material, reducing any overestimation of biodegradation occurring in phase 2. Further testing is required to assert whether this is a viable solution and to determine how long phase 1 must be extended, e.g. perhaps until the degradation curve falls below $100 \%$.

The advantage of the two-step design is that it is a good compromise between test complexity and minimizing unspecific (excess) gas formation caused by the surface-activity of the test substance. For the most part, this has been achieved with the majority of results consistent with the literature.

\section{Methods}

\section{Participating laboratories}

The test protocol for the AnBUSDiC test, as detailed in [5], was shared with seven laboratories who had experience in undertaking biodegradation studies. These were (in alphabetical order);

- Department of Experimental Toxicology and Ecology BASF SE, Ludwigshafen (Germany)

- Dr. Noack-Laboratorium, Hildesheim, (Germany)

- Fraunhofer UMSICHT, Oberhausen (Germany)

- Harlan Laboratories, Derby (UK)

- Hydrotox GmbH, Freiburg (Germany)

- Organic Waste Systems (OWS) NV, Gent (Belgium)

- Stadtentwässerungsbetrieb Landeshauptstadt, Düsseldorf (Germany)
Each laboratory was instructed to follow the modified AnBUSDiC test method. Information about the test substances (e.g. empirical formula), the detailed test procedure and the source and preparation of the inoculum are provided elsewhere [5]. Any deviations from the protocol due to equipment limitations and/or differences were discussed and agreed beforehand.

\section{Basis of the method}

A mixture of anaerobic fermentative and methanogenic bacteria is involved in the anaerobic decomposition process such as is usually encountered in the digestion tower of municipal sewage plants. The different microorganisms decompose the organic carbon compounds via various intermediate stages to form the ultimate degradation products methane $\left(\mathrm{CH}_{4}\right)$ and carbon dioxide $\left(\mathrm{CO}_{2}\right)$. The community of all these microorganisms forms the digested sludge in the anaerobic sludge treatment stage in the WWTPs.

In order to determine the anaerobic degradation, an exactly defined amount of the substance is incubated together with sludge inoculum at $35^{\circ} \mathrm{C} \pm 1^{\circ} \mathrm{C}$. The sludge inoculum consists of digested sludge to which a certain amount of raw sludge (raw sludge is usually a mixture of $40 \%$ sludge from the pre-sedimentation and $60 \%$ sludge from the final sedimentation in WWTPs) has been added in order to 'vitalize' it, i.e. to obtain a basic sludge gas development. The addition of raw sludge is part of the original DIN 38414, part 8. When the suitability of the method for determining the biodegradability of surfactants was evaluated, this step was retained, as the basic sludge gas development enables any possible inhibitory effects caused by the test substance to be observed. The sludge gas production is measured using a eudiometer. The net gas production of the test batch is obtained by subtracting the volume of gas produced by the control batch. The gas law is then used to convert the net gas volume into the molar gas amount, taking pressure and temperature into account. The degree of degradation of the test substance is then determined by the rule of three from the measured molar gas amount and the organic carbon provided by the test substance and used in the test.

\section{Preparing the sample}

As surfactants are a quite heterogeneous group of chemicals with regard to structure, charge and molecular weight, they have different physicochemical properties. Some are solid, others are liquid at room temperature, and some are even pasty and therefore difficult to handle. To assure that a sample of the test substance is homogeneous, it may be necessary to gently warm it up whilst shaking before removing an aliquot for carbon analysis or for addition to the degradation test. The 
organic carbon content of the test substance is determined as the TOC $(\mathrm{g} / \mathrm{g})$ (according to DIN EN 13137 in a double determination) and a known amount of the test substance is used in the test. A test substance concentration of 200 to $800 \mathrm{mg}$ active substance is used for the first addition (i.e. 150 to $500 \mathrm{mg} / \mathrm{L}$ TOC). After the net gas evolution in the test sample has come to an end (i.e. the degradation kinetic of the test sample and the control are more or less identical), a second addition of another 200 to $800 \mathrm{mg}$ active substance is made directly to the test system with gentle mixing.

\section{Carrying out the test}

The determination of the biodegradability of the test substance is made by determining the difference in the amounts of digester gas produced by the test batch and the control batch. The test and control batches are dosed with identical aliquots of the sludge inoculum.

In general, the DIN 38414, part 8 (1985) test is more tolerant to higher test substance concentrations than the ECETOC test, which is caused by the higher inoculum density. As a rule of thumb 150 to $500 \mathrm{mg} / \mathrm{L} \mathrm{TOC}$ of the test substance is a good starting concentration for most surfactants, but for some surfactants even $1,500 \mathrm{mg} / \mathrm{L}$ TOC was not found to be inhibitory.

The test solutions $(500 \mathrm{ml}$ sludge inoculum and sludge inoculum with test substance) are placed in the incubation bottles of the eudiometers as described above. These are then sealed with a ground joint stopper and gently homogenized without any air entrainment. The incubation bottles are then fitted with the eudiometer attachments and treated in parallel by placing them in a water bath held at $35^{\circ} \mathrm{C} \pm 1^{\circ} \mathrm{C}$. The eudiometers are filled to the zero mark with the barrier liquid with the stopcock open. All tests and controls are carried out at least as double determinations. Each water bath contains one control batch and two test batches as a double determination.

It is assumed that any unspecific digester gas evolution caused by the surface activity of the test substance takes place within the first 3 weeks. Therefore, after the initial degradation reaction has reached the plateau phase (usually at about day 21), another aliquot of the test substance (with or without the toxicity control) is added. To avoid oxygen entering the system, this is done, e.g. under a nitrogen atmosphere. Verification of the anaerobic status of the test system at the end of the test could be done using an oxygen indicator, a redox dye, resazurin. However, as in the OECD 311 method [10]; it is not straightforward to confirm the color of the dye easily without filtration of sludge under nitrogen, which requires further work.

Further it should be kept in mind that the correct TOC reference value defining $100 \%$ degradation of the added test substance(s) is used for the calculation of the degradation rate of the second phase (see evaluation).

The batches are swirled around once per day. The gas volumes are initially read off and recorded on a daily basis; subsequently the reading intervals can be lengthened to several days as is necessary. For reading the evolved digester gas volume, the meniscus of the liquid collection bottle and the meniscus of the liquid inside the eudiometer should be aligned. All readings are noted in the test protocol. After each reading, the barrier liquid in the eudiometer is readjusted to the zero mark. In addition, the water bath and room temperatures are measured and recorded on a daily basis (this is important for the conversion of the gas volumes to molar amounts).

The daily determined gas amounts are added together for each batch, but separately for phase 1 and phase 2 (both starting at zero) and presented as gas production curves and, after appropriate conversion, also as degradation curves.

The tests are continued until the net gas production has reached the plateau phase or the degradation has reached more than $80 \%$ of the theoretical amount.

\section{Ring test conditions}

All participating laboratories ran the six test substances (five surfactants + control) through the test procedure as expected for the ring test. The TOC content of each test substance was pre-determined and provided to the participating laboratories. This information allowed the laboratories to create suitable concentrations of each test substance in the range of 200 to $800 \mathrm{mg} / \mathrm{L}$ or 150 to 500 $\mathrm{mg} / \mathrm{L}$ organic carbon (OC). Laboratories were able to refine the dosing and individually determine suitable concentrations of test substances, according to their experimental set up. Any changes to the experimental set up due to equipment availability or otherwise, were preagreed beforehand. Each laboratory sourced their own wastewater treatment plant inocula from a nearby domestic WWTP. This was then prepared in the appropriate way according to the test procedure outlined.

Six test substances representing a variety of surfactant types including a positive control (glucose) were tested anonymously by the laboratories. Details of the substances are as follows:

- Glucose (CAS 50-99-7) - the positive reference compound, not surface active

- isoC9-11 alcohol ethoxylate [7EO] (CAS 78330-20 -8) - non-ionic surfactant (\#23 DID list [11])

- Linear C12-18 alcohol ethoxylate [7EO] (CAS 68213-23-0) - non-ionic surfactant (\#35 DID list)

- C12 alkyl sulfonate (CAS 151-21-3) - anionic surfactant (\#5 DID list) 
- Cocoamidopropyl Betaine (CAS 61789-40-0) amphoteric surfactant (\#61 DID list)

- C10-13 linear alkylbenzene sulfonate [LAS] (CAS 68411-30-3) - anionic surfactant (\#1 DID list)

\section{Competing interests}

The authors declare that they have no competing interests.

\section{Authors' contributions}

$C E, A B, G C, J D F, C H, D S, K T$ and $A W$ elaborated the testing scheme and monitored the ring test. TA and CE drafted the manuscript. All authors read and approved the final manuscript.

\section{Authors' information}

$C E, A B, G C, J D F, C H, D S, K T$ and $A W$ are Members of the ERASM Anaerobic Task Force. TA is an Ecotoxicologist (Contractor) working for Shell Health Environment and Product Health (EPH).

\section{Acknowledgements}

The authors thank the ERASM Technical Committee (Brussels) for their financial support of this study and all of the participating laboratories that generated test data using the AnBUSDiC method.

\section{Author details}

'Shell Health, Shell Technology Centre - Thornton, P O Box 1, Chester CH1 $3 \mathrm{SH}$, Cheshire, UK. ${ }^{2}$ European Chemical Industry Council, avenue E van Nieuwenhuyse 4, Brussels 1160, Belgium. ${ }^{3}$ Sasol Italy S. p A, Stabilimento di Augusta (SR), C da Marcellino s. n. C P 119, Augusta, SR 96011, Italy. ${ }^{4}$ Cepsa Quimica S A, Fabrica de Ouneta Mayorga Apdo Correos 40, San Roque, Cádiz 11360, Spain. ${ }^{5}$ Sasol Germany GmbH, Building 1033/PB02, Paul-Baumann-Str. 1, Marl 45772, Germany. ${ }^{6}$ Procter \& Gamble, Brussels Innovation Center (BIC), Temselaan 100, Strombeek-Bever 1853, Belgium. 'BASF SE, GUP/PA - Z470, Ludwigshafen 67056, Germany. ${ }^{8}$ BASF Personal Care and Nutrition GmbH, Henkelstr 67, Dusseldorf 40589, Germany.

\section{Received: 2 April 2013 Accepted: 6 August 2013}

Published: 15 August 2013

\section{References}

1. Berna JL, Cassani G, Hager C-D, Rehman N, Lopez I, Schowanek D, Steber J, Taeger K, Wind T: Anaerobic biodegradation of surfactants - scientific review. Tenside Surf Det 2007, 44(6):312-347.

2. OECD Guideline for the Testing of Chemicals: 314: Simulation Tests to Assess the Biodegradability of Chemicals Discharged in Wastewater. Paris: Organization for Economic Co-operation Development; 2008.

3. Fraunhofer Institut UMSICHT: Anaerobic biodegradability of detergent surfactants, anaerobic biodegradability of detergent surfactants. Final Report. Oberhausen, Germany: Fraunhofer Institut UMSICHT; 2003 [http://ec.europa. eu/enterprise/sectors/chemicals/documents/competitiveness/anaerobic_en. $\mathrm{htm}]$

4. DIN 38414, part 8: German Standard Methods for the Examination of Water, Waste Water and Sludge; Sludge and Sediments (Group S): Determination of the Amenability to Anaerobic Digestion (S 8). Berlin: Deutsches Institut Fur Normung E.V. (German National Standard); 1985.

5. Bendt $T$, Willing $A: A$ new method to determine the anaerobic degradability of surfactants: the AnBUSDiC test. Environ Sci Eur 2012 24:28.

6. Atlas RM: Microbial degradation of petroleum hydrocarbons: an environmental perspective. Microbiological reviews 1981, 45(1):180-209.

7. Dorn PB, Wong DCL, Ye J, Martin VA: Chemical properties affecting the environmental performance of synthetic-based drilling fluids for the gulf of Mexico, Report SPE 142008. In SPE Americas E\&P Health, Safety, Security and Environmental Conference. Houston, Texas, USA; Richardson, Texas 75080: Society of Petroleum Engineers; 2011.

8. Lara-Martin PA, Gomez-Parra A, Sanz JL, Gonzales-Mazo E: Anaerobic degradation pathway of linear alkylbenzene sulfonates (LAS) in sulfate-reducing marine sediments. Environ Sci Technol 2010, 44(5):1670-1676.

9. Pagga U, Beimborn DB: Anaerobic biodegradation test for organic compounds. Chemosphere 1993, 27(8):1499-1509.
10. OECD Guidelines for the Testing of Chemicals: 311: Anaerobic Biodegradability of Organic Compounds in Digested Sludge: by Measurement of Gas Production. Paris: Organization for Economic Co-operation Development; 2006.

11. EU: Detergents Ingredients List: 2007. [http://ec.europa.eu/environment/ ecolabel/ecolabelled_products/categories/pdf/did_list/didlist_part_a_en.pdf]

doi:10.1186/2190-4715-25-23

Cite this article as: Eadsforth et al:: Ring testing of the AnBUSDiC test; a new screening method for the anaerobic degradability of surfactants. Environmental Sciences Europe 2013 25:23.

\section{Submit your manuscript to a SpringerOpen ${ }^{\circ}$ journal and benefit from:}

- Convenient online submission

- Rigorous peer review

- Immediate publication on acceptance

- Open access: articles freely available online

High visibility within the field

- Retaining the copyright to your article

Submit your next manuscript at $>$ springeropen.com 